\title{
Preserving Polarization through an Intrinsic Depolarizing Resonance with a Partial Snake at the AGS $^{1}$
}

\author{
H.Huang*, L. Ahrens*, M. Bai*, K.A. Brown*, J.W. Glenn*, A.U. Luccio*, \\ W.W. MacKay*, C. Montag*, V. Ptitsyn*, V. Ranjbar ${ }^{\dagger}$, T. Roser*, H. \\ Spinka** $^{* *}$ N. Tsoupas*, D.G. Underwood ${ }^{* *}$ and K. Zeno* \\ ${ }^{*}$ C-A Department, Brookhaven National Laboratory, Upton, NY 11973, USA \\ ${ }^{\dagger}$ Physics Department, Indiana University, Bloomington, IN 47505, USA \\ ** Argonne National Laboratory, Argonne, IL 60439, USA
}

\begin{abstract}
An 11.4\% partial Siberian snake was used to successfully accelerate polarized protons through a strong intrinsic depolarizing spin resonance in the AGS. No noticeable depolarization was observed. This opens up the possibility of using a $20 \%$ partial Siberian snake in the AGS to overcome all weak and strong spin resonances.
\end{abstract}

\section{INTRODUCTION}

Acceleration of polarized proton beams to high energy in circular accelerators is difficult due to numerous depolarizing resonances. It is particularly difficult in the medium energy range since the limited available straight sections in the existing synchrotrons make it very hard to install a full Siberian snake [1] to correct all types of depolarizing spin resonances. During acceleration, a depolarizing resonance is crossed whenever the spin precession frequency equals the frequency with which spin-perturbing magnetic fields are encountered. In the presence of the vertical dipole guide field in an accelerator, the spin precesses $G \gamma$ times per orbit revolution [2], where $G=(g-2) / 2=1.7928$ is the gyromagnetic anomaly of the proton, and $\gamma$ is the Lorentz factor. The number of precessions per revolution is called the spin tune $v_{s p}$ and is equal to $G \gamma$ in this case.

There are three main types of depolarizing resonances: imperfection resonances, which are driven by magnet misalignments; intrinsic resonances, driven by the vertical betatron motion through quadrupoles; and coupling resonances, caused by the vertical motion with horizontal betatron frequency due to linear coupling [3]. The resonance condition for an imperfection resonance is $v_{s p}=n$, where $n$ is an integer. The resonance condition for an intrinsic resonance is $v_{s p}=n P \pm v_{y}$, where $n$ is an integer, $P=12$ is the super-periodicity of the AGS, and $v_{y}$ is the vertical betatron tune. The resonance condition for a coupling spin resonance is $v_{s p}=n \pm v_{x}$, where $v_{x}$ is the horizontal betatron tune; it is only important in the vicinity of a strong intrinsic resonance.

\footnotetext{
1 This work was supported by the DOE of USA.
}

CP675, Spin 2002: 15th Int'l. Spin Physics Symposium and Workshop on Polarized Electron Sources and Polarimeters, edited by Y. I. Makdisi, A. U. Luccio, and W. W. MacKay (C) 2003 American Institute of Physics 0-7354-0136-5/03/\$20.00 
At the AGS, a 5\% partial Siberian snake [5] has been used to overcome the imperfection resonances [6], and an ac dipole has been used to overcome strong intrinsic resonances [7]. For a ring with a partial snake with strength $s$, the spin tune $v_{s p}$ is given by

$$
\cos \pi v_{s p}=\cos \frac{s \pi}{2} \cos G \gamma \pi
$$

where $s=1$ would correspond to a full snake which rotates the spin by $180^{\circ}$. When $s$ is small, the spin tune is nearly equal to $G \gamma$ except when $G \gamma$ equals an integer $n$, where the spin tune $v_{s p}$ is shifted away from the integer by $\pm s / 2$. Thus, the partial snake creates a gap in the spin tune at all integers. Since the spin tune never equals an integer, the imperfection resonance condition is never satisfied. Thus the partial snake can overcome all imperfection resonances, provided that the resonance strengths are much smaller than the spin-tune gap created by the partial snake.

By adiabatically exciting a vertical coherent betatron oscillation using a single ac dipole magnet, an artificial spin resonance is excited. If the resonance location is chosen near an intrinsic spin resonance, the spin motion will be dominated by the ac dipole resonance, and full spin flip can be achieved [7]. However, the ac dipole technique only works for strong intrinsic resonances, since it relies on the strength of the intrinsic resonances to induce a strong enough artificial resonance. With a strong enough partial snake, the spin tune gap can be increased to allow placing the betatron tune inside the gap so the intrinsic resonance conditions can also be avoided. Simulations showed that for the first intrinsic resonance at $0+v_{y}$, a $10 \%$ partial snake would be strong enough. In a recent experiment at the AGS this was successfully demonstrated. This paper will present the results and discussions.

\section{EXPERIMENT SETUP}

The polarized $\mathrm{H}^{-}$beam from the optically pumped polarized ion source (OPPIS) [8] was accelerated through a radio frequency quadrupole and the $200 \mathrm{MeV}$ LINAC. The beam polarization at $200 \mathrm{MeV}$ was measured with elastic scattering from a carbon fiber target. During the study, the polarization measured by the $200 \mathrm{MeV}$ polarimeter was $(66 \pm 0.5) \%$. The beam was then strip-injected and accelerated in the AGS Booster up to $1.5 \mathrm{GeV}$ kinetic energy or $G \gamma=4.7$. The vertical betatron tune of the AGS Booster was chosen to be 4.9 in order to avoid crossing the intrinsic resonance $G \gamma=0+v_{y}$ in the Booster. The imperfection resonances at $G \gamma=3,4$ were corrected by harmonic orbit correctors. Only one bunch of the twelve if buckets in the AGS was filled, and the beam intensity varied between $1.3-1.7 \times 10^{11}$ protons per fill. The polarized proton beam was accelerated up to $G \gamma=12.5$ or $5.6 \mathrm{GeV}$ kinetic energy passing through just one intrinsic resonance located at $G \gamma=0+v_{y}$. The resonance crossing speed $\alpha=\frac{d(G \gamma)}{d \theta}$ was $2.4 \times 10^{-5}$. Polarization was measured at $G \gamma=12.5$ during an approximately one second flattop after the partial snake was ramped to zero. At $G \gamma=0+v_{y}$, the solenoid can generate a $25 \%$ partial snake. At the same time, the solenoidal field will generate significant coupling, which will cause sizeable depolarization. In addition, such 


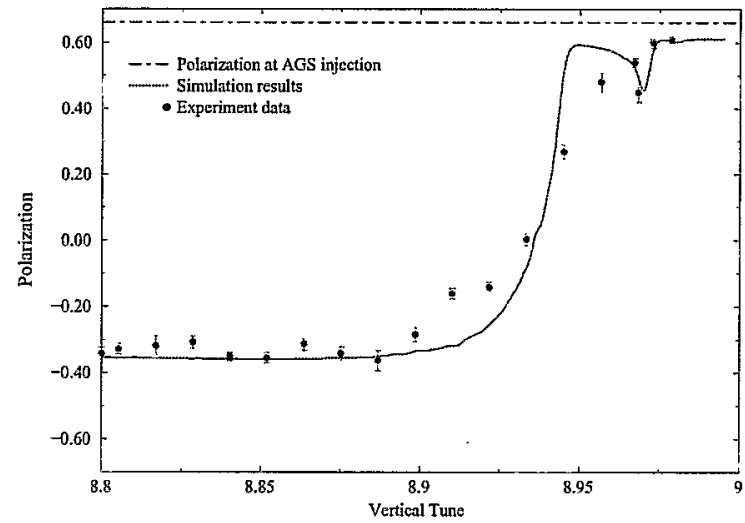

FIGURE 1. The measured vertical polarization as a function of the vertical betatron tune for an $11.4 \%$ partial snake. The dots are measured polarization, and the error bars only represent the statistical errors. The solid straight line indicates the polarization level measured at the end of the LINAC. Since the two imperfection resonances in the Booster have been corrected by harmonic orbit correctors, this is also the polarization at AGS injection. The solid curve shows the simulation results.

a strong snake will tilt the stable spin direction away from vertical by $12.5^{\circ}$, reducing the measurable vertical polarization component. An $11.4 \%$ partial snake was chosen as a compromise between obtaining a large enough spin tune gap and minimizing the coupling effects. The AGS partial snake was turned on before injection at $6 \%$ and ramped up to $11.4 \%$ for the first intrinsic resonance at $0+v_{y}$. The orbit was carefully corrected to maintain stable beam as the vertical betatron tune was moved as high as 8.98. During the experiment, the horizontal tune was kept at 8.54 , while the beam polarization was measured as a function of the vertical betatron tune.

\section{RESULTS AND DISCUSSION}

The experimental data and simulation results are plotted in Fig.1. The simulation is a combination of a DEPOL [9] calculation and a tracking model with two overlapping resonances: one located at $G \gamma=9$ generated by the partial snake and the intrinsic resonance at $G \gamma=0+v_{y}$, which changes its location when the vertical betatron tune changes. The strength of the intrinsic resonance was determined from beam size measurements using the AGS ionization profile monitor(IPM). A \pm 0.004 vertical tune spread was included in the simulation. The vertical tunes were not measured in the tune window $v_{y}=8.9$ to 8.96. A fitting of measured tunes vs. set tunes outside the window was done. Inside the window vertical tunes were derived from the set values based on the fitting. The strengths of the coupling resonances located at $G \gamma=17-v_{x}, 0+v_{x}, 18-v_{x}$, and $1+v_{x}$ were calculated from the extended DEPOL [9] program. Since the coupling resonances are separated from the other two resonances, they can be treated independently. When the intrinsic and imperfection resonances do not overlap $\left(v_{y} \sim 8.85\right)$, the resonance at $G \gamma=9$ will flip spin completely while the intrinsic resonance at $G \gamma=0+v_{y}$ 
will flip spin partially and cause depolarization. Note the polarization is getting slightly lower when the vertical betatron tune moves closer to the horizontal betatron tune; this is due to stronger coupling resonances. When the two resonances are very close, such as $v_{y}=8.98$, the intrinsic resonance is overpowered by the resonance at $G \gamma=9$. Beam essentially just experiences one resonance at $G \gamma=9$, and full spin flip is observed. When the two resonances are a modest distance apart, such as for $v_{y}>8.90$, the two resonances interfere with each other, and $G \gamma=9$ resonance gradually dominates when the vertical betatron tune goes higher. The turning point is $v_{y} \sim 8.94$ because an $11.4 \%$ partial snake generates a 0.057 gap on each side of the integer. In general, most data points agree well with the simulation. The remaining discrepancies for data points between $v_{y}=8.9$ and 8.96 could be explained with different beam size or different vertical betatron tune, but there were no beam size and vertical tune measurements for these data points.

Both experiment and simulation show a polarization dip close to $v_{y}=8.97$. This is caused by a snake resonance [10]. Even when the intrinsic resonance condition can not be met for $v_{y}>8.95$, depolarization can occur from resonance conditions extended over many turns if the intrinsic resonance is very strong. This happens when the following condition is met

$$
\Delta v_{y}=\frac{k \pm v_{s p}}{n},
$$

where $\Delta v_{y}$ is the fractional part of vertical betatron tune, $n$ and $k$ are integers, and $n$ is called the snake resonance order. With an $11.4 \%$ partial snake, the spin tune is close to 0.057 for $G \gamma \sim 9$. The polarization dip then corresponds to the second order snake resonance $(n=2)$. With the given acceleration rate and intrinsic resonance strength, snake resonances higher than second order do not show a significant effect. The existence of the snake resonance reduced the usable tune space and the betatron tune had to be carefully chosen to avoid depolarization.

At the vertical betatron tune of 8.98 , the difference between the polarization at injection and polarization measured after $0+v_{y}$ is due to the spin mismatch at injection and some additional depolarization from coupling resonances. If spin matching were achieved at AGS injection and the linear coupling were eliminated, the scheme could provide full spin flip through the intrinsic resonance. It also would work for weak intrinsic resonances, such as $G \gamma=24 \pm v_{y}, 48-v_{y}$.

\section{OUTLOOK}

In conclusion, we have demonstrated for the first time that an $11.4 \%$ partial snake can effectively overcome an intrinsic depolarizing resonance when the vertical betatron tune is put close to an integer. The critical element of this operation is to maintain beam stability with betatron tune close to an integer. For AGS, this method has several advantages. First, it works for both strong and weak intrinsic resonances. Currently, there is no effective way to overcome the weak intrinsic resonances in the AGS. Second, if the coupling of a new snake could be reduced, the strength of coupling resonances could also be reduced. Or, if both horizontal and vertical tunes could be put into the gap, both intrinsic and coupling resonances could be avoided. Simulation shows that a $20 \%$ 


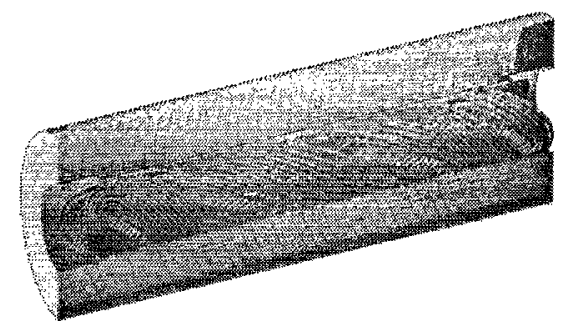

FIGURE 2. The design of superconducting snake magnet. It is 2.6 meters long and the beam pipe is 15 $\mathrm{cm}$ by $15 \mathrm{~cm}$.

partial snake is needed for the strongest intrinsic resonance at $36+v_{y}$. This is beyond the capability of the existing solenoidal partial snake. Furthermore, the solenoidal field is the main source of coupling, which causes coupling resonances in the vicinity of strong intrinsic resonances. The better choice would be a helical dipole magnet as has been used in RHIC polarized proton operation. With the constraint of 10-foot AGS straight section, the required field can only be achieved by a super-conducting magnet. With compensating coils, the coupling from the new snake can be greatly reduced. The design of a super-conducting helical AGS snake with strength on the order of $20 \%$ to replace the current solenoidal AGS partial snake has already begun. A sketch of the design is shown in Fig. 2. With the given field, the snake strength at AGS injection would be about $24 \%$. At injection energy, the tune shifts are of the order of 0.2 units, and the beta functions fluctuate throughout the ring up to values around $100 \mathrm{~m}$ instead of the matched maximum of about $22 \mathrm{~m}$. A solution has been found by E. Courant [11] to eliminate the coupling and beta function mismatch caused by the snake. In addition, such a strong snake in the AGS will tilt the stable spin direction away from vertical significantly. Study of the spin matching at injection and extraction from the AGS is also underway to ensure the best polarization transfer efficiency at AGS injection and extraction.

\section{REFERENCES}

1. Ya.S. Derbenev and A.M. Kondratenko, Part. Accel. 8, 115 (1978).

2. L.H. Thomas, Philos. Mag. 3, 1 (1927); V. Bargmann, L. Michel, and V.L. Telegdi, Phys. Rev. Lett. 2. 435 (1959).

3. H. Huang, T. Roser, A. Luccio, Proc. of 1997 IEEE PAC, Vancouver, May, 1997, p.2538.

4. M. Froissart and R. Stora, Nucl. Instrum. Meth. 7, 297(1960).

5. T. Roser, in Proceedings of the 8th International Symposium on High-Energy Spin Physics, Minneapolis, 1988, AIP Conf. Proc. No 187 (AIP, New York,1989), p.1442.

6. H. Huang, et al., Phys. Rev. Lett. 73, 2982 (1994).

7. M. Bai, et al., Phys. Rev. Lett. 80, 4673 (1998).

8. A. Zelenski, et al., in Proceedings of the 9 th International Conference on Ion Sources, Rev. Sci. Inst., Vol.73, No.2, p.888 (2002).

9. V. Ranjbar, et al., Proc. of 2001 IEEE PAC, Chicago, June, 2001, p. 3177; E.D. Courant and R.D. Ruth, BNL report 51270, (1980).

10. S.Y. Lee and S. Tepikian, Phys. Rev. Lett. 56, 1635 (1986).

11. E. Courant, these proceedings. 